\title{
On the Occurrence of Naked Singularities in General Relativity *
}

\author{
P. Yodzis, H.-J. Seifert, and H. Müller zum Hagen \\ I. Institut für Theoretische Physik, Universität Hamburg, Hamburg, \\ Federal Republic of Germany
}

Received April 27; in revised form June 4, 1973

\begin{abstract}
It is shown that spherically symmetric collapse can lead to singularities which are not hidden within "black holes".
\end{abstract}

\section{Introduction}

It is widely believed that, in the framework of general relativity, gravitational collapse inevitably leads to singularities which are hidden within "black holes"; that "naked singularities" are forbidden by some basic principles of relativity physics. We need hardly point out the fundamental importance of this conjecture (but will do so anyway): should it turn out to be false, then gravitational collapse would force far reaching revisions of present physical theory, since this would be tantamount to a disastrous breakdown of general relativity.

The conjecture (like most conjectures about gravitational collapse) is based to a large extent upon properties of the (spherically symmetric) Schwarzschild-Kruskal vacuum solution, with its singularity hidden behind the familiar horizon. Implicit in this way of thinking is the assumption that the presence of matter does not appreciably alter the character of the singularity. This is not necessarily a sensible extrapolation.

We shall show here that in fact even spherically symmetric collapse can lead to naked singularities.

Our results do not exclude the possibility that some suitably sharpened version of the conjecture may hold (there is some discussion about this below), but they certainly indicate that the conjecture cannot be taken for granted and must be scrutinized much more closely than it has been up to now.

Let us begin by defining some terms. As usual, we restrict attention to spacetimes which contain only a single object ${ }^{1}$, and can therefore

* Work supported by the Deutsche Forschungsgemeinschaft.

${ }^{1}$ In physical terms, this means that the collapsing object is assumed sufficiently far away from everything else in the universe so that everything else can be ignored. 
reasonably be assumed to be weakly asymptotically simple. We say that this object is undergoing gravitational collapse if 1) the spacetime contains a regular ("initial") spacelike hypersurface on which the energymomentum tensor has connected compact support and all physical quantities are well behaved, 2) there exists a causal curve within the matter $^{2}$ which is nonextendible into the future and has finite affine length. As first principles we require that Einstein's field equations are fulfilled, with an energy-momentum tensor that satisfies the dominant energy condition [1], and that the spacetime is stably causal. We say that the singularity is naked if there exists a causal curve ${ }^{3}$ with one end on $\mathscr{I}^{+}$and the other end "on the singularity".

Let us briefly contrast naked singularities with hidden ones. Recall that the horizon in a weakly asymptotically simple spacetime is defined as the boundary of the past of $\mathscr{I}^{+}$; that is, it is the boundary of the set of events in spacetime which can communicate with the asymptotic "flat" region. In the case of a hidden singularity, no nonextendible incomplete causal curve can lie entirely in the region which can communicate with $\mathscr{I}^{+}$; roughly speaking, "the singularity is hidden behind the horizon". The mere existence of a nonempty horizon does not, however, in itself preclude the existence of a naked singularity (see, for instance, the examples in Section II below).

There are of course many well known static solutions which have naked singularities, but their physical significance is far from clear, and they are in any case excluded from the present discussion by condition 1) of our definition of gravitational collapse.

In Section II we show that naked singularities can occur for collapsing spherically symmetric dust clouds. In Section III we show that even if there are nonzero pressures, naked singularities can still occur. Section IV contains some conclusions and remarks.

\section{Collapsing Dust Clouds}

The line element for a spherically symmetric dust cloud with "Newtonian" initial conditions [2] is, written in comoving coordinates (the radial coordinate is fixed as follows: the ball of coordinate radius $r$ contains $r$ particles of dust),

$$
d s^{2}=-d t^{2}+R^{2}\left(d \theta^{2}+\sin ^{2} \theta d \varphi^{2}\right)+R_{0}^{\prime 2} B^{2} A^{-\frac{2}{3}} d r^{2},
$$

${ }^{2}$ By "within the matter" we of course mean: within the region where the energymomentum tensor is nonzero. From the physical point of view, one is particularly interested in the case where some invariantly defined physical quantities (for instance the energy density) tend toward infinity along this curve; this is the behaviour in the examples we give below.

${ }^{3}$ In our examples, there exists such a causal curve which is also a geodesic. 
where' denotes partial derivative with respect to $r$,

$$
\begin{aligned}
& R(r, t):=R_{0}(r) A^{\frac{2}{3}} \\
& A(r, t):=1-\frac{3}{2}\left(2 \mu r / R_{0}^{3}\right)^{\frac{1}{2}} t \\
& B(r, t):=1-\frac{3}{2}\left(2 \mu r / R_{0}^{3}\right)^{\frac{1}{2}}\left(R_{0} / 3 r R_{0}^{\prime}\right) t
\end{aligned}
$$

and the positive constant $\mu$ is the rest mass of a dust particle. This holds for $r \leqq b$, where $r=b>0$ is the boundary of the dust cloud; the junction conditions to a (Schwarzschild) vacuum exterior are automatically fulfilled. The energy-momentum tensor is $T^{a b}=\varrho \delta_{4}^{a} \delta_{4}^{b}$, with the energy density $\varrho$ given by

$$
\varrho=\mu\left(4 \pi R_{0}^{2} R_{0}^{\prime} A B\right)^{-1} .
$$

The Ricci scalar is of course proportional to the energy density in this case.

Let us choose

$$
R_{0}(r)^{3}= \begin{cases}f(r), & r \in[0, b / 2] \\ k(r / b)^{\varepsilon} \exp (a \cdot \cos (\pi r / b)), & r \in[b / 2, b],\end{cases}
$$

where the monotonically increasing function $f(r)$ is such that $R_{0}{ }^{3}$ is of class $C^{3}$ for $r \in[0, b], f \sim r$ in a neighbourhood of $r=0$, and $k, \varepsilon$, and $a$ are positive constants. The exact form of $f(r)$ will not be of any interest to us.

In order to have a finite initial energy density [Eq. (3)], we must have $a<\varepsilon \cdot M$, where $M:=\max \{K \mid 1-K x \cdot \sin x \geqq 0, x \in[\pi / 2, \pi]\} ; M$ exists and is a finite positive number.

We shall also require that the initial radius of the cloud is greater than its Schwarzschild radius. Since the total mass of the cloud is $\mu b$, this means that if we set

$$
N:=2 \mu b / R_{0}(b),
$$

then $N$ must be smaller than 1 . By the way, this last equation enables us to express $k$ as a function of $\mu, b, \varepsilon, a$, and $N$.

As the time increases, $\varrho$ increases, approaching infinity as the hypersurface $B=0$ is approached. This hypersurface can therefore be regarded as a singular boundary for the spacetime (we shall give this singular boundary a causal structure below). Let us fix $b$ and $\mu$ since these quantities are of no significance for the qualitative behaviour of the spacetime. We then have a family of spacetimes parametrized by $\varepsilon, a$, and $N$. We shall show that each of these spacetimes belongs to one of the following three subfamilies:

I. The singularity is hidden behind a horizon.

II. The event horizon of the spacetime (which is just the event horizon of the vacuum exterior maximally extended into the interior 
of the cloud) terminates on the singularity, as, for instance, in the causal diagram (Fig. 1). In general, part of the singularity extends below the point of intersection with the horizon and can therefore communicate with $\mathscr{I}^{+}$: the singularity is naked.

III. The horizon does not cross the surface of the matter, as shown in Fig. 3: Again the singularity is naked. This subfamily has a particularly interesting causal structure, which will be discussed below.

We shall now show how these properties arise. Observe first that if we describe the singular boundary by writing $t=t_{s}(r)$, this function has the following behaviour: 1) it is positive and finite everywhere on the singular boundary, 2) it has a single minimum in the interval $(b / 2, b]$, $3)$ the value of $t_{s}$ at this minimum can be made arbitrarily small (if we set $a=\varepsilon M$, the minimum value of $t_{s}$ would be precisely 0 ).

The time at which the singularity reaches the surface of the matter is $t_{s}(b)$; let $t_{h}$ be the time at which the surface of the matter crosses the horizon. Then $t_{s}(b)$ is given by $B\left(b, t_{s}(b)\right)=0$, with $B(r, t)$ as in Eq. (2); and $t_{h}$ is given by $R\left(b, t_{h}\right)=2 \mu b$, with $R(r, t)$ as in Eq. (2) (which is just the familiar vacuum horizon condition that the total mass $\mu b$ should be equal to half of the curvature radius $R$ ). One finds, using also the definition of $N$, that

$$
t_{s}(b)=\frac{4}{3} \mu b N^{-\frac{3}{2}} \varepsilon, \quad t_{h}=\frac{4}{3} \mu b N^{-\frac{3}{2}}\left(1-N^{\frac{3}{2}}\right) .
$$

Plainly, for any choice of $a$ and $N$, if $\varepsilon<1-N^{\frac{3}{2}}$ the spacetime belongs to subfamily III.

Suppose instead that $\zeta:=\varepsilon\left(1-N^{\frac{3}{2}}\right)^{-1}>1$. Obviously, if $a$ is sufficiently small the spacetime belongs to subfamily I. Now let us obtain a sufficient condition for the spacetime to belong to subfamily II. Since, by choosing $a$ sufficiently large, we can bring the earliest point of the singularity in the region $b / 2<r \leqq b$ arbitrarily close to $t=0$; such a sufficient condition is clearly that, in the region $S$ specified by $b / 2<r \leqq b, 0<t<\min \left(t_{s}(r), t_{h}\right)$, the "slope" of the horizon (that is to say, $d r / d t$ evaluated on a radial curve lying in the horizon) is bounded above by $2 t_{h} / b$. From Eq. (1), one sees that this condition can be written as

$$
R_{0}^{\prime} B A^{-\frac{1}{3}}<2 t_{h} / b
$$

throughout $S$. A careful examination of $R_{0}^{\prime} B A^{-\frac{1}{3}}$ shows that, throughout $S$,

$$
R_{0}^{\prime} B A^{-\frac{1}{3}}<\frac{4}{3} \zeta \mu \exp \left(\zeta\left(1-N^{\frac{3}{2}}\right) M / 3\right) \cdot\left(1-N^{\frac{3}{2}}\right) N^{-\frac{3}{2}}
$$

independent of the choice of $a$. Therefore, if $\zeta-1$ and $1-N^{\frac{3}{2}}$ are sufficiently small and $a$ is sufficiently large, the required inequality holds and the spacetime belongs to subfamily II. 
Now let us look at the maximal vacuum extensions of these spacetimes. We shall display the extensions for subfamilies II and III only (Figs. 1-3).

These pictures represent 2 -surfaces $\theta=$ constant, $\varphi=$ constant; that is, they correspond to the $r-t$ plane. This plane is the orbit space of the rotation group (which is the isometry group of our spacetimes), since each point in it represents a 2-sphere on which the rotation group acts transitively. Thus the timelike curve which is the center of spherical symmetry is represented in our pictures as a boundary line (labelled in the pictures as "center line").

Our diagrams correspond to the $r$ - $t$ plane, but they are not exactly the $r$ - $t$ plane. Firstly, this plane has been distorted in such a way that all null directions lying in it have slope \pm 1 . Secondly, these are "Penrose pictures": null infinity is represented as a finite boundary, depicted in our diagrams by a double line. All singularities are represented by crosshatched lines. Since the projection into the orbit space of any causal curve in the spacetime is again a causal curve, and since the null curves in our pictures are simply straight lines with slope \pm 1 , this sort of diagram is very well suited for the eludication of causal structure.

Figure 1 shows the maximal vacuum extension for subfamily II, with $\varepsilon \geqq 1$. The curve $C$ represents the initial hypersurface $t=0$. The horizon consists of two disjoint null surfaces; note that these surfaces terminate on the singularity. Region $a$ is causally isolated behind the horizon, and region $d$ is causally isolated within the dust. Observers in region $c$ can see the singularity.

For subfamily II, with $\varepsilon<1$, the extension is a little more complicated. The singularity within the matter in this case cannot terminate on the $R=0$ singularity of the Kruskal vacuum exterior, because $R$ [in Eq. (1)] for our interior solution is nonzero for all $r \geqq b / 2$. Therefore the spacetime can be extended across the curve $\overline{P Q}$ : this is done by identifying the curve $\overline{P Q}$ with one side of the curve $\overline{P^{\prime} Q^{\prime}}$ in the vacuum Kruskal manifold (which has mass $\mu b$ ) pictured in Fig. 2. The other side of the curve $\overline{P^{\prime} Q^{\prime}}$ is identified with one side of a similar curve in yet another vacuum Kruskal manifold (again with mass $\mu b$ ), and so on. The point $P$ is singular in the sense that the curvature approaches infinity as $P$ is approached from some directions, while $P^{\prime}$ is only a branch point. Otherwise said: the maximally extended orbit space cannot be conformally embedded in the Euclidean 2-plane, but only in the logarithmic Riemann surface. In Fig. 2 (and also in Fig. 3) two sheets of the Riemann surface are depicted.

The regions $a, c$, and $d$ have the same properties as for the case $\varepsilon \geqq 1$. Region $e$ can communicate with some points in the future of the initial surface $C$. 


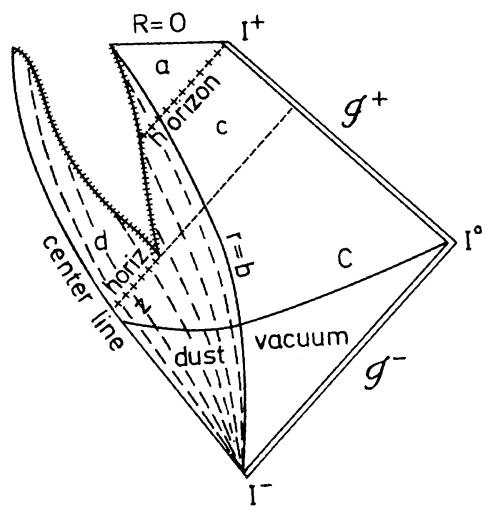

Fig. 1
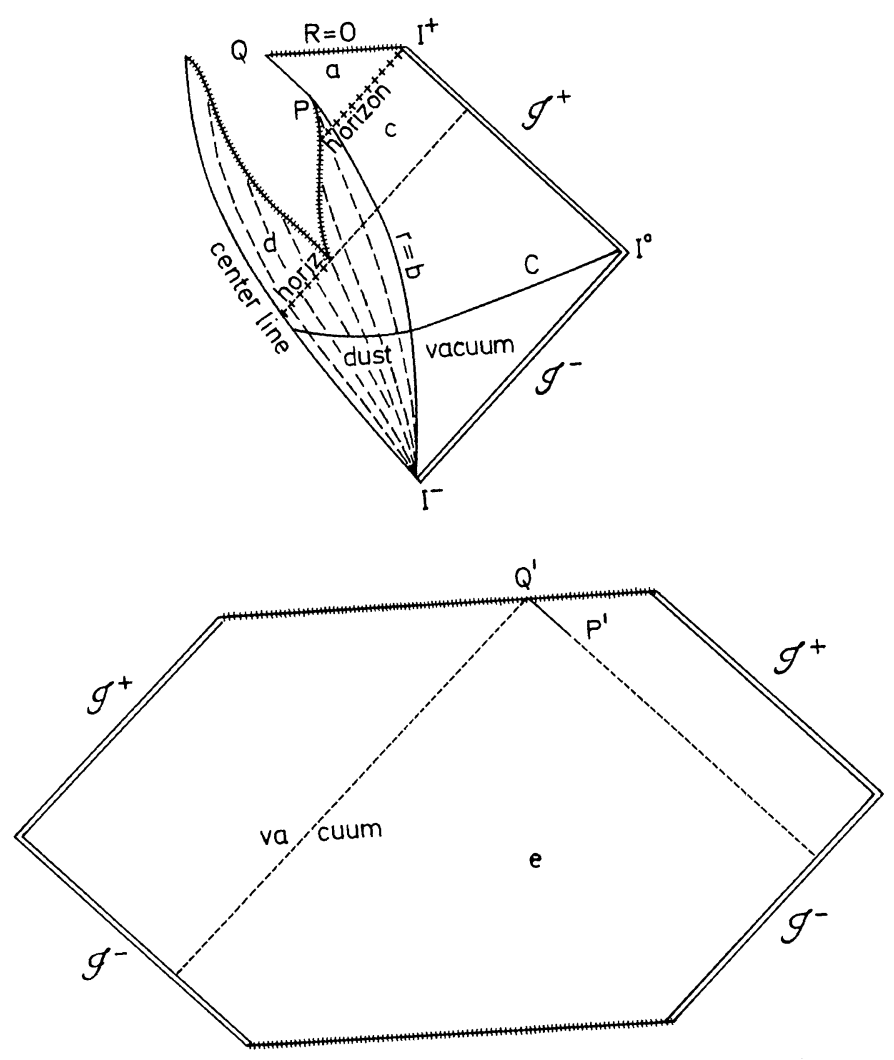

Fig. 2 

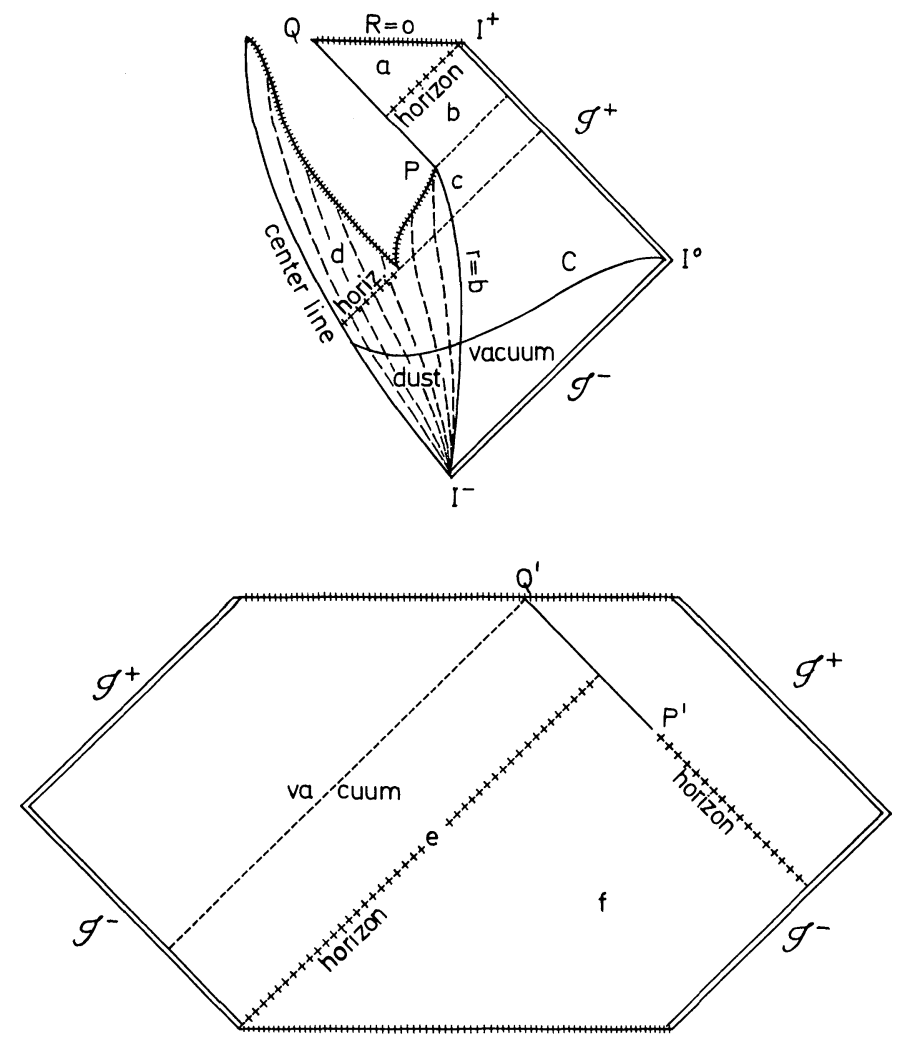

Fig. 3

For subfamily III, $\varepsilon$ is always less than 1 and the causal structure is always as shown in Fig. 3. The curves $C$ and $\overline{P Q}$ and the regions $a, c, d$, and $e$ have the same meanings as in the cases just discussed. The new feature in subfamily III is the occurrence of the regions $f$ and $b$. Events in region $f$ can communicate, across $\overline{P^{\prime} Q^{\prime}}$ via region $b$, with that $\mathscr{I}^{+}$ which lies in the future of $C$ : thus, the spacetime contains a "white hole".

Returning to the general solution (1), (2), (3), one can characterize the singularities of collapsing dust clouds with "Newtonian" initial conditions as follows ${ }^{4}$. For each value of $r$, the singular boundary occurs at the value of $t$ given by $A(r, t)=0$ if $R_{0}(r) / 3 r R_{0}^{\prime}(r)<1$, and at the value of $t$ given by $B(r, t)=0$ if $R_{0}(r) / 3 r R_{0}^{\prime}(r) \geqq 1$. This can be seen from Eq. (3).

4 A similar characterisation can be given for non-Newtonian initial conditions, but it cannot be stated so concisely. Incidentally, for "Newtonian" initial conditions, the spacetime is regular into the past. 
The limit of the metric as the singular boundary is approached gives the causal behaviour of the singular boundary, regarded as a causal boundary. From Eq. (1), one sees that points of the singular boundary for which $R_{0}(r) / 3 r R_{0}^{\prime}(r)<1$ are spacelike, and points of the singular boundary for which $R_{0} / 3 r R_{0}^{\prime} \geqq 1$ are timelike or null. Points of the singular boundary which are spacelike cannot communicate with $\mathscr{I}^{+}$.

A spacelike point of the singular boundary can be enclosed in a spacelike 2-sphere (in the spacetime) of arbitrarily small (metric) radius.

The nonspacelike points of the singular boundary correspond to a "crossing" of two $r=$ constant "shells" of dust, or (in geometric terms) to the formation of a Kruskal-like "throat" in a $t=$ constant hypersurface.

The collapsing dust spacetimes with naked singularities violate none of the first principles set out above. They are, however, utterly unrealistic, since they contain no pressure. It appears that naked singularities can be ruled out (if they can be ruled out at all) only by invoking some "second principles" which dictate that the energy-momentum tensor should be "realistic". Unfortunately, it is much more difficult to state second principles than it is to state first principles, and it is also more difficult to maintain one's certitude about second principles. A minimal second principle would surely be that the pressures should be nonzero; let us demand also that they should be positive, though this need not always hold (one need only think of a hard crust). We have not yet been able to show precisely how the properties of dust cloud singularities outlined above are altered by the presence of nonzero pressures, but the discussion in the next section does show this much: even if one adds the second principle we have just stated to our first principles, this still is not enough to exclude naked singularities.

\section{Collapse with Pressure}

Let us write the line element of a spherically symmetric spacetime in the form

$$
d s^{2}=-e^{\gamma} d t^{2}+r^{2}\left(d \theta^{2}+\sin ^{2} \theta d \varphi^{2}\right)+e^{\alpha} d r^{2},
$$

where $\alpha$ and $\gamma$ are functions of $r$ and $t$. As it is well known, the "curvature coordinate" $r$ is not always an admissible coordinate (it fails to be admissible if the spacetime contains a Kruskal-type "throat"), but in the spacetimes discussed in this section it is in fact admissible. Consider first a static perfect fluid, with equation of state

$$
p_{0}(r)=\zeta \cdot \varrho_{0}(r)-\hat{p}, \quad \zeta \in(0,1 / 3), \quad \hat{p}=\text { const }>0
$$


For such an equation of state there exists a bounded solution of Einstein's field equations, with boundary at $r=R$, say, and with $\varrho_{0}(r)$ uniquely fixed, for any given central density $\varrho_{0}(0)$ [3]. (In the present section, all quantities with a subscript ${ }_{0}$ refer to this static perfect fluid solution.)

Now let us make the following ansatz:

$$
\begin{aligned}
& \varrho:=-T_{4}^{4}=a(t) b(r)+\varrho_{0}(r) \\
& p:=T_{1}^{1}=\zeta a(t) b(r)+\zeta \cdot \varrho_{0}(r)-\hat{p}
\end{aligned}
$$

where

$$
0 \leqq a(t) \leqq 1 ; \quad 0 \leqq \dot{a}(t) \leqq 1 ; \quad a(t) \equiv 0 \quad \text { for } \quad t \leqq t_{0},
$$

$b(r)$ defined on $(0, R] ;$ for $r \rightarrow 0: b \rightarrow+\infty, b(r) / \ln \frac{r}{R}$, $b^{\prime}(r) \cdot r$, and $b^{\prime \prime}(r) \cdot r^{2}$ are bounded. $b(r)$ is $C^{2}$ on $(0, R]$.

$$
b(R)=0=b^{\prime}(R) ; \int_{0}^{R} \hat{r}^{2} b(\hat{r}) d \hat{r}=0 .
$$

As shown for instance by Synge [4], if one is given the quantities $\varrho(r, t)$ and $p(r, t)$, one can then construct a complete solution of Einstein's equations having $T_{4}^{4}=-\varrho$ and $T_{1}^{1}=p$, by direct integration. We shall demand that some physical principles are satisfied, which will lead to certain further restrictions on $a(t)$; these further restrictions are compatible with one another and are consistent with the restrictions (5a) already stated above. Throughout this section,' means $\frac{\partial}{\partial r}$ and ' means $\frac{\partial}{\partial t}$.

The metric coefficients are given by (we set the coupling constant $\kappa=1)$

$$
\begin{aligned}
e^{-\alpha} & =1-\frac{1}{r} \int_{0}^{r} \hat{r}^{2} \varrho(\hat{r}) d \hat{r}=1-\frac{2 m_{0}}{r}-\frac{a}{r} \int_{0}^{r} \hat{r}^{2} b(\hat{r}) d \hat{r} \\
\gamma & =-\alpha+\int_{0}^{r} \hat{r} e^{\alpha}(p+\varrho) d \hat{r}
\end{aligned}
$$

where

$$
2 m_{0}:=\int_{0}^{r} \hat{r}^{2} \varrho_{0}(\hat{r}) d \hat{r} .
$$


Note that if $a(t)$ is sufficiently small, then $\exp (-\alpha)$ is positive and finite for all $r \leqq R$, and $\gamma$ is finite for all $r \leqq R$.

The component $T_{4}^{1}$ of the energy-momentum tensor is given, for $r \leqq R$, by

$$
T_{4}^{1}=-\frac{\dot{a}}{r^{2}} \int_{0}^{r} \hat{r}^{2} b(\hat{r}) d \hat{r}
$$

From the conditions $(5 \mathrm{c})$ it follows that $T_{1}^{1}(R)=0$ and $T_{4}^{1}(R)=0$, which implies that this solution can be matched to a Schwarzschild vacuum exterior across the surface $r=R$.

It can be shown that in any interval $\left(0, r_{0}\right], r_{0}<R$ :

$$
\begin{aligned}
\alpha^{\prime} & =-\left(\ln e^{-\alpha}\right)^{\prime}=-e^{\alpha}\left(e^{-\alpha}\right)^{\prime}=r \cdot \ln \frac{r}{R} \cdot f_{1}, \\
\gamma^{\prime} & =-\alpha^{\prime}+r \cdot e^{\alpha}(p+\varrho)=r \cdot \ln \frac{r}{R} \cdot f_{2}, \\
\dot{\alpha} & =-e^{\alpha}\left(e^{-\alpha}\right)^{\cdot}=\dot{a} \cdot r \cdot f_{3}, \\
\dot{\gamma} & =\dot{a} \cdot r \cdot f_{4}, \\
-\dot{T}_{4}^{4} & =\dot{a} b, \\
T_{4}^{1} & =\dot{a} f_{5}, \\
T_{1}^{1 \prime} & =\zeta a b^{\prime}+\zeta \varrho_{0}^{\prime},
\end{aligned}
$$

where $f_{1}, f_{2}, f_{3}, f_{4}$ are functions of $r$ and $t$ and $f_{5}$ is a function of $r$, such that $f_{i}$ and $f_{i}^{\prime} \cdot r$ are bounded for all $r \in\left(0, r_{0}\right]$ and for all $t$, for each $i=1, \ldots, 5$. To show this one uses the fact that $a(t)$ is bounded for all $t$, and also the fact that

$$
\frac{1}{r^{3} \ln \frac{r}{R}} \int_{0}^{r} \hat{r}^{2} b(\hat{r}) d \hat{r}
$$

is bounded near $r=0$. This last assertion follows from ( $5 \mathrm{~b})$ along with the observation that, by L'Hospital's rule,

$$
\lim _{r \rightarrow 0} \frac{1}{r^{3} \ln \frac{r}{R}} \int_{0}^{r} \hat{r}^{2} b(\hat{r}) d \hat{r}=\frac{b(r)}{\ln \frac{r}{R}} \cdot\left(3+\frac{1}{\ln \frac{r}{R}}\right)^{-1},
$$


Since $r \cdot \ln \frac{r}{R}$ is bounded, so is

$$
\frac{1}{r^{2}} \int_{0}^{r} \hat{r}^{2} b(\hat{r}) d \hat{r}
$$

Finally, we may note that a similar argument shows that the quantities

$$
\frac{1}{r}\left(\frac{2 m_{0}}{r}\right)^{\prime} \text { and }\left(\frac{2 m_{0}}{r}\right)^{\prime \prime}
$$

are bounded on $(0, R]$.

Note that, since $\dot{a}$ can be made arbitrarily small, so can $T_{4}^{1}$. This means that our quantities $\varrho$ and $p$ can be made as close as we like to the two eigenvalues of the energy-momentum tensor which correspond to the energy density and the principal radial pressure. Since, from Eqs. (4), the quantities $\varrho$ and $p$ satisfy the "equation of state" $p=\zeta \varrho-\hat{p}$, it follows that we can come as close as we like to a situation in which the radial principal pressure and the energy density are related by such an equation of state. Therefore, at least so far as the energy density and the radial pressure are concerned, our first and second principles are satisfied, and in an eminently reasonable way.

It remains only to examine $T_{2}^{2}$, which is given by

$$
\begin{aligned}
T_{2}^{2}= & T_{1}^{1}+\frac{r}{2} T_{1}^{1 \prime}+\frac{r}{4} \gamma^{\prime}\left(T_{1}^{1}-T_{4}^{4}\right)+\frac{r}{2} \dot{T}_{1}^{4}-\frac{r}{4} e^{\alpha-\gamma}(\dot{\alpha}+\dot{\gamma}) T_{4}^{1} \\
= & p+r a b^{\prime} \zeta / 2+\varrho_{0}^{\prime} r \zeta / 2+r^{2} \ln \frac{r}{R}(1+\zeta) b a f_{2} / 4 \\
& +r^{2} \ln \frac{r}{R}\left(\varrho_{0}+p_{0}\right) f_{2} / 4-r e^{\alpha-\gamma}\left[2 \ddot{a}+r\left(3 f_{3}-f_{4}\right) \dot{a}^{2}\right] f_{5} / 4 .
\end{aligned}
$$

Note first that $T_{2}^{2}$ vanishes at $r=R$ since $T_{2}^{2}(R)$ is time independent, and for $t \leqq t_{0}$ the energy-momentum tensor is that of a static perfect fluid. Note further that $T_{2}^{2}-p$ is of the form $a f_{6}+\dot{a} f_{7}+\ddot{a} f_{8}$ (of course $T_{2}^{2 \prime}-p^{\prime}$ is $\left.a f_{6}^{\prime}+\dot{a} f_{7}^{\prime}+\ddot{a} f_{8}^{\prime}\right)$ where $f_{6}, f_{7}$, and $f_{8}$ are bounded everywhere inside the matter, and $f_{6}^{\prime}, f_{7}^{\prime}$, and $f_{8}^{\prime}$ are bounded everywhere inside the matter except in an arbitrarily small neighbourhood of $r=0$. Therefore, by choosing $a, \dot{a}$, and $\ddot{a}$ sufficiently small, we can fit $T_{2}^{2}$ into an arbitrary uniform $C^{0}$ neighbourhood of $p$, and if we exclude a neighbourhood of $r=0$, we can fit $T_{2}^{2}$ into an arbitrary uniform $C^{1}$ neighbourhood of $p$. This means in particular, since $p^{\prime}$ is bounded away from zero in a neighbourhood of $r=R$ (because $p^{\prime}(R)=p_{0}^{\prime}(R)>0$ ) that $T_{2}^{2}$ is positive everywhere inside the matter. Moreover, since $T_{2}^{2}$ can be made arbitrarily close to $p$, the dominant energy condition is fulfilled, and what is more, our matter is almost (in the sense just explained) a perfect fluid. 
From the form of $\varrho$, Eq. (4), it is clear that the line $r=0, t>t_{0}$ is singular in the sense that as this line is approached, the density and pressure tend toward infinity. However, the metric coefficients have a $C^{1}$ extension onto this singular line, and the extended $\gamma$ is finite everywhere, whence the singularity is timelike and the (extended) spacetime contains no horizons. That is: the singularity is naked.

There is one further detail to be dealt with: we have not shown that our conditions $(5 \mathrm{~b}, \mathrm{c})$ on the function $b(r)$ are compatible. In fact, they are compatible since, for instance, the function

$$
b(r)=-\left[\ln \frac{r}{R}+\frac{5}{6}\left(\frac{r}{R}\right)^{2}-\frac{8}{3} \frac{r}{R}+\frac{11}{6}\right]
$$

satisfies them.

The question may well arise in the reader's mind: what is the essential physical mechanism for the formation of this remarkable singularity? It is the following. Our matter is almost a perfect fluid, but not quite. The small difference between $T_{1}^{1}$ and $T_{2}^{2}$ has been arranged mathematically in just such a way as to "squeeze" the matter into a (naked) singularity.

It is conceivable that one could obtain, by an iteration process, solutions in which an exact equation of state is satisfied. It seems to us quite likely that in such solutions the boundary of the object would have to collapse, and it is also possible that the causal character of the singularity would be altered; whether these two processes would conspire to hide the singularity is impossible to say at this point.

The reader will note that there is a sudden jump in $\varrho(r=0)$ at the time $t_{0}$, from a constant finite to an infinite value. This is not in any sense a characteristic of naked singularities, but only reflects the particularly simple form of our ansatz (4). This sudden jump can be avoided within the framework of the present method, but only at the expense of making our equations more complicated. Likewise, our requirement that $T_{4}^{1}$ should be "small" has no fundamental significance, but only enables us to easily interpret the quantities $\varrho$ and $p$. Again, the property that the boundary of the matter remains fixed at $r=R$ has no fundamental significance, but is a matter of mathematical convenience. Even in the present approach a moving boundary could be incorporated, but would only add complication, rather than insight.

Is the above solution (really: set of solutions) somehow very peculiar and special, so much so that the set of spacetimes with naked singularities is of "measure zero" in the space of all spherically symmetric spacetimes? This is a very difficult and subtle question, whose answer depends, for instance, on what one means by "measure zero". For the moment, we 
can only remark that, even within the context of our present method, one can construct solutions which are "almost" perfect fluids and have naked singularities, with rather weak restrictions on the ansatz from which one starts.

One can have a naked singularity for any ("almost" obeyed) equation of state, so long as $p$ is a monotonically increasing function of $\varrho$, is dominated by $\varrho$, and reaches zero at a positive value of $\varrho$. One then has the following freedom in choosing the behaviour at small $r$ of the ansatz for $\varrho$ : 1) if $p$ remains finite as $\varrho$ approaches infinity, then any choice of $\varrho$ which is bounded above by $r^{-1}$ in a neighbourhood of $r=0$ is allowed; 2) if $p$ approaches infinity as $\varrho$ approaches infinity, but $p$ is bounded above by $\varrho^{\varepsilon}(0<\varepsilon<1)$ for large $\varrho$, then any choice of $\varrho$ which is bounded above by $(-\ln r)^{1 / \varepsilon}$ in a neighbourhood of $r=0$ is allowed; 3) for any other equation of state, any choice of $\varrho$ which is bounded above by $-\ln r$ in a neighbourhood of $r=0$ is allowed.

\section{Concluding Remarks}

It should be clear from the above that if there exists a cogent statement which rules out naked singularities, it must be worded with some care. In our view, there are two directions along which one might try to approach such a statement.

The first one is to consider the question of stability. We have shown that first principles, and probably any reasonable second principles, allow naked singularities. However, we have not shown that this property is stable with respect to, say, initial data and equations of state.

The second approach would be insist that the second principles we have taken into account thus far are too weak. In particular, one could demand that an exact equation of state, subject to some restrictions, is obeyed; we have already discussed this possibility somewhat in Section III. However, especially in the presence of timelike singularities, it seems problematical to decide what is really a "reasonable" equation of state, for one can conceive of the possibility that there could be formidable radiation terms to be taken into account, so that a reasonable equation of state may look at the first glance very strange.

It is an open question whether there exists a cogent statement excluding naked singularities. The resolution of this question is likely to involve some very interesting considerations, and to produce some physical insight.

Acknowledgement. We thank Wolfgang Kundt and Bernd Schmidt for stimulating discussions. 


\title{
References
}

1. Hawking, S. W.: Commun. math. Phys. 18, 301-306 (1970)

2. Misner, C. W.: Gravitational collapse. In: (Ed.): Chrétien, M., Deser, S., Goldstein, J.): Astrophysics and General Relativity. New York: Gordon and Breach 1969

3. Schmidt, B. G.: Kugelsymmetrische statische Materielösungen der Einsteinschen Feldgleichungen (Diplomarbeit). Hamburg 1966

4. Synge, J. L.: Relativity: The general theory. Amsterdam: North-Holland Publ. Comp. 1960

\author{
H. Müller zum Hagen \\ I. Institut für Theoretische Physik \\ der Universität \\ D-2000 Hamburg 36 \\ Jungiusstr. 9 \\ Federal Republic of Germany
}

Note added in proof. Meanwhile, we have extended the results of Section II as follows (H. Müller zum Hagen, P. Yodzis, and H.-J. Seifert, "On the occurance of Naked Singularities in General Relativity II", preprint U. Hamburg, August 1973): we have shown that naked "shell crossing" singularities can occur in the spherically symmetric collapse of perfect fluids, for a large family of equations of state in which the pressure has an (arbitrarily large) upper bound, and that this behaviour is stable with respect to spherically symmetric perturbations of the initial data, as well as with respect to perturbations of the equation of state. 